\title{
Insuficiência renal crônica: representações sociais de pacientes com e sem depressão
}

\author{
Fabrycianne Gonçalves Costa - Universidade Federal da Paraíba, João Pessoa, Brasil \\ Maria da Penha de Lima Coutinho - Universidade Federal da Paraíba, João Pessoa, Brasil \\ Inayara Oliveira de Santana - Universidade Federal do Recôncavo da Babia, Santo Antônio de Jesus, Brasil
}

\begin{abstract}
Resumo
Este estudo objetivou apreender as representações sociais acerca da insuficiência renal crônica (IRC) elaboradas por pacientes com e sem depressão no contexto da hemodiálise. Participaram do estudo 26 pacientes nefrológicos, com idades entre 22 e 73 anos $(M=44,19 ; D P=11,83)$, que responderam a uma entrevista semiestruturada e à Escala Hospitalar de Ansiedade e Depressão (HADS). Os resultados evidenciaram que $20 \%$ apresentaram sintomas depressivos. Os pacientes com a síndrome depressiva apontaram, em suas representações acerca da IRC, o impacto do diagnóstico em suas vidas, que foi permeado por sentimentos de medo da morte e recusa em iniciar o tratamento. Os pacientes sem a depressão destacaram elementos associados ao significado positivo do tratamento e ao apoio recebido pela família. Os resultados apontam para a importância de uma abordagem interdisciplinar que enfoque o suporte psicossociológico e nutricional no tratamento da doença renal.

Palavras-chave: Insuficiência renal crônica; Hemodiálise; ALCESTE; Representações sociais; Depressão.
\end{abstract}

\section{Chronic renal failure: social representations of depression and non-depression patients}

\begin{abstract}
In this research, we sought to learn about the social representations on chronic renal failure (CRF) and the treatment elaborated by depressed or non-depressed patients in the context of hemodialysis. As many as 26 nephrology patients participated, with ages ranging from 22 and 73 years old $(M=44,19 ; \mathrm{SD}=11,83)$, who responded to a semi-structured interview and to the Hospital Anxiety and Depression Scale (HADS). The results showed that $20 \%$ had depression symptoms. The depression-syndrome patients pointed out, in their representations on the CRF, the diagnosis' impact over their lives, which was permeated by feelings such as fear of dying and refusal to initiate the treatment. The non-depression patients highlighted elements associated both with the treatment's positive results and with the support received by the family. Outcomes indicate the relevance of an interdisciplinary approach that focuses on the psycho-social and nutritional support in the treatment of renal diseases.

Keywords: Chronic renal failure; Hemodialysis; ALCESTE; Social representations; Depression.
\end{abstract}

Insuficiencia renal crónica: representaciones sociales de pacientes con y sin depresión

\begin{abstract}
Resumen
Este estudio tuvo como objetivo aprehender las representaciones sociales en cuanto a la insuficiencia renal crónica (IRC) elaboradas por pacientes con y sin depresión en el contexto de hemodiálisis. Participaron 26 pacientes nefrológicos, con edades entre 22 y 73 años $(M=44,19 ; D P=11,83)$, que contestaron a una entrevista semiestructurada y la Escala Hospitalaria de Ansiedad y Depresión (HAD). Se observó que un $20 \%$ de la muestra presentaron síntomas depresivos. Los pacientes con síndrome depresiva señalaron, en sus representaciones en cuanto a la IRC, el impacto del diagnóstico en sus vidas, que se permearon por sentimientos de miedo de la muerte y recusa en iniciar el tratamiento. Los pacientes sin depresión señalaron elementos asociados al significado positivo del tratamiento y al apoyo recibido por la familia. Los resultados señalan la importancia de un abordaje interdisciplinaria que enfoca el soporte sicosociológico y nutricional en el tratamiento de la enfermedad renal.

Palabras-clave: Insuficiencia renal crónica; Hemodiálisis; ALCESTE; Representaciones sociales; Depresión.
\end{abstract}

\section{Introdução}

A insuficiência renal crônica (IRC) é a fase mais avançada da doença renal. Consiste na perda progressiva, irreversível e multifatorial, da capacidade dos rins em manter os equilíbrios metabólicos e hidroeletrolíticos, gerando alterações nos diversos sistemas do organismo (Smeltzer \& Bare, 2009).

$\mathrm{Nas}$ fases iniciais da IRC, quando as manifestações clínicas e laboratoriais são mínimas ou ausentes, o diagnóstico pode ser sugerido pela associação de manifestações inespecíficas (fadiga, anorexia, emagrecimento,

Disponivel em www.scielo.br prurido, náusea ou hemólise, hipertensão e edema). Outras alterações fisiológicas frequentes são: a poliúria (urinar acima de 2,5 litros por dia associado a um aumento na frequência urinária, que pode ser noturna) e a hematúria (existência de sangue na urina) (Ribeiro e cols., 2008). Com o progresso da doença, ocorre o acúmulo de substâncias no sangue e algumas toxinas não eliminadas podem afetar o organismo de diversas maneiras, resultando em um quadro de uremia, devido ao acúmulo de ureia.

Por ser uma doença assintomática, os indivíduos desconhecem a sua existência até o quadro clínico se 
apresentar bastante avançado e necessitar, com urgência, de tratamento para substituir a função renal (Romão Junior, 2007; Smeltzer \& Bare, 2009). O tratamento mais comumente adotado nesses casos é a hemodiálise. Trata-se de um processo mecânico e extracorpóreo, que consiste na remoção de substâncias tóxicas e do excesso de líquido do organismo. Geralmente, esse tratamento é realizado em uma unidade hospitalar, três vezes por semana, em sessões que duram três ou quatro horas. Além desses cuidados, a pessoa em tratamento deve seguir dietas e ingerir medicamentos que auxiliam na reestruturação do funcionamento renal (Thomas \& Alchieri, 2005).

Nesse contexto, o paciente é levado a conviver diariamente com uma doença incurável, que o obriga a uma forma de tratamento dolorosa, de longa duração e que provoca, juntamente com a evolução da doença e suas complicações, maiores limitações e alterações de grande impacto. As repercussões da doença ocorrem tanto na própria vida do doente quanto na do seu grupo familiar, afetando as várias dimensões do ser humano, sejam elas de ordem física, psicológica, econômica ou social (Lima \& Gualda, 2000; Ramos, Queiroz \& Jorge, 2008).

Como explicam Ferreira e Anes (2010), deve-se levar em conta a constante exposição a fatores adversos inerentes à terapêutica da síndrome renal, como o tempo gasto nas sessões de hemodiálise, as constantes consultas médicas, os exames laboratoriais, as dietas e a expectativa de transplante. Associados à frequente permanência em ambientes hospitalares, todos esses fatores têm contribuído para o surgimento da depressão nessa população.

Estudos desenvolvidos por Nifa e Rudnicki (2010), Chilcot, Wellsted, Silva-Gane e Farrington (2008) têm demonstrado que a depressão parece ser o transtorno psíquico mais frequente nos pacientes em tratamento de hemodiálise. Pesquisas como as de Garcia, Veiga e Motta (2010), apontaram para uma prevalência de $68 \%$ de depressão em pacientes renais, enquanto Costa, Coutinho, Melo e Oliveira (no prelo) constataram 56,3\%, e Finger, Pasqualotto, Marcon, Medeiros, Abruzzi Junior e May (2011) 44\%. Além destes, Cukor, Peterson, Cohen e Kimmel (2006) encontraram uma prevalência de $42 \%$. Semelhantemente, Kimmel, Cohen e Peterson (2008) assinalaram para uma variação de 30\% e, conforme Almeida (2003), a incidência da depressão pode variar entre 10 e $20 \%$, dependendo dos instrumentos utilizados.
Ao longo do século XX, observou-se um gradativo aumento de interesse na compreensão do binômio saúde-doença, considerando-se os aspectos biopsicossociais que perpassam esse fenômeno. Em consequência, nas últimas três décadas desse mesmo século, a associação entre a depressão e as doenças crônicas, a exemplo da IRC, passou a ser identificada e estudada, aproveitando-se as contribuições da psicologia da saúde e da psicologia social (Kimmel e cols., 2008; Nifa \& Rudnicki, 2010).

São diversos os significados que passam pelo imaginário das pessoas afetadas pela IRC, indo desde o impacto do diagnóstico, associado ao reconhecimento da gravidade da doença e do tratamento, até as suas consequências, como os efeitos medicamentosos e os limites nos hábitos alimentares e na vida social (Ramos e cols., 2008). Pereira e Guedes (2009) ressaltam que as mudanças nos hábitos de vida em face da decorrência da doença geram dificuldades associadas à ausência de experiências que proporcionam prazer, à privação do trabalho, às dificuldades financeiras, à incapacidade física para desempenhar as atividades cotidianas, à necessidade do deslocamento para outra cidade para realizar as sessões de hemodiálise. Referem-se, ainda, as preocupações associadas à preservação do funcionamento das fístulas, controle hídrico e restrições alimentares. Em geral, essas situações provocam dúvidas, insegurança, medo, angústia e sofrimento quanto à cura e à possibilidade de viver.

Destarte, a IRC pode gerar desordens emocionais que, quando sobrepostos à condição física, afetam diretamente a qualidade de vida dessas pessoas (Ramos e cols., 2008). Nesse sentido, faz-se importante compreender como os indivíduos acometidos por uma doença crônica simbolizam a experiência produzida por tal realidade, uma vez que as representações sociais contribuem para direcionar os comportamentos, comunicações e práticas cotidianas, incluindo as reações ao tratamento (Coutinho \& Saraiva, 2013).

Diante dessas premissas, este estudo objetivou rastrear a depressão nos pacientes em tratamento de hemodiálise, bem como apreender as representações sociais acerca da insuficiência renal crônica elaboradas por pacientes com e sem depressão no contexto da hemodiálise. Como eixo norteador, utilizou-se a abordagem psicossocial fundamentada na Teoria das Representações Sociais (TRS).

Conforme Moscovici (2011), as representações sociais (RS) de um grupo de pertença constituem o resultado de um processo de transformação daquilo 
que é não familiar nem conhecido em algo familiar e particular. Portanto, uma função básica das RS é a integração do novo, o que é conseguido por meio de dois processos interligados, a ancoragem e a objetivação. Nesses processos, são levadas em consideração a memória e as conclusões preestabelecidas, de modo a formar um esquema conceptual ou de referência. Dessa forma, as informações não são incorporadas de modo automático à vida dos indivíduos.

A ancoragem transfere o desconhecido para o esquema de referência, por meio da comparação e da interpretação; por sua vez, o processo de objetivação reproduz o desconhecido, entre o que é visível e tangível. Assim, surge uma RS ante um novo objeto, por meio da materialização de uma entidade abstrata, que foi ancorada pela classificação e pela nomeação (Moscovici, 2011).

Ter acesso às representações sociais de um objeto social é tentar compreender as formas que os indivíduos utilizam para criar, transformar e interpretar uma problemática vinculada a sua realidade. Significa também tentar conhecer seus pensamentos, sentimentos, percepções e experiências de vida compartilhada, de acordo com a classe social a que pertencem e com as instituições às quais estão vinculados (Coutinho, 2005). O acesso às representações sociais formuladas por pacientes com e sem depressão no contexto da hemodiálise pode possibilitar compreender como esses indivíduos interpretam essa nova realidade de vida, permeada pela doença e pelo tratamento. Dessa maneira, constitui um material que pode ser utilizado pela comunidade científica, na elaboração de planos de tratamento que levem em consideração os aspectos psicossociais (afetivos, cognitivos) e não apenas físicos (hemodiálise e dieta alimentar).

\section{Método}

Trata-se de uma pesquisa descritiva, de campo, com cunho quantitativo e qualitativo, apoiada em uma abordagem psicossociológica.

\section{Participantes}

A investigação foi desenvolvida em três instituições hospitalares, localizadas no município de João Pessoa/PB, que disponibilizam o tratamento da hemodiálise para adultos e mantêm convênio com o Sistema Único de Saúde (SUS). Participaram 26 pacientes nefrológicos, com idades entre 22 e 73 anos $(M=44,19$; $\mathrm{DP}=11,83)$, sendo, em sua maioria, do sexo feminino
$(57,7 \%)$, casados $(84,6 \%)$ e com ensino fundamental completo $(57,7 \%)$.

Os critérios de inclusão da amostra foram: (i) estar em tratamento de hemodiálise há pelo menos três meses; (ii) concordar em responder os instrumentos de coleta de dados, firmando o Termo de Consentimento Livre e Esclarecido; (iii) ter idade mínima de 18 anos; e (iv) possuir capacidade de compreensão adequada para responder os instrumentos.

\section{Instrumentos}

Foi utilizada a técnica de entrevista semiestruturada, que consistiu num roteiro com perguntas básicas e abertas sobre a IRC, contemplando quatro temas gerais: 1. Gostaria que o(a) senhor(a) falasse tudo o que conhece acerca da IRC; 2 . O que o(a) senhor(a) sentiu ao receber o diagnóstico, quais foram os seus pensamentos, sentimentos e comportamentos?; 3. O que o tratamento para a IRC significa na sua vida?; 4. Quais as dificuldades que $\mathrm{o}(\mathrm{a})$ senhor(a) tem encontrado para seguir o tratamento?

Para a caracterização da amostra, foi utilizado um questionário sociodemográfico, com questões relacionadas à idade, ao sexo, à escolaridade e ao estado civil. Para verificar a presença de sintomas depressivos nos pacientes, utilizou-se a Escala Hospitalar de Ansiedade e Depressão (HADS), adaptada à população brasileira por Botega, Bio, Zomignani, Garcia e Pereira (1995) e Castro e cols.(2006). Essa escala possui 14 itens, dos quais sete são voltados para a avaliação da ansiedade (HADS-A) e sete para a depressão (HADS-D), apresentando os respectivos coeficientes de alfa de Cronbach, 0,68 e 0,77 . Cada item pode ser pontuado de zero a três, compondo uma pontuação de $0 \pm 21$, sendo recomendável "8" como ponto de corte Botega e cols., 1995 (Marcolino e cols., 2007). Neste estudo, utilizou-se a subescala que se refere à depressão (HADS-D), como instrumento de screening. O tempo total de aplicação dos instrumentos foi, em média, de 30 minutos.

\section{Procedimentos éticos e de coleta de dados}

A pesquisa foi aprovada pelo Comitê de Ética em Pesquisa, que funciona no Hospital Universitário Lauro Wanderley, da Universidade Federal da Paraíba, sob o protocolo de $n^{\circ} 392 / 11$. Durante a sua realização, foram respeitadas todas as normas éticas advindas da Resolução 196/96, do Conselho Nacional de Saúde (Brasil, 1996).

Após o recebimento do parecer favorável, foi feito um contato com a diretoria das instituições escolhidas, 
onde as pesquisadoras se apresentaram com uma carta contendo os objetivos e a importância do desenvolvimento da pesquisa. Em seguida, os dados foram coletados individualmente, nas mesmas instituições hospitalares em que os pacientes realizam o tratamento da hemodiálise. Todos os participantes assinaram um Termo de Consentimento Livre e Esclarecido, bem como foram informados do caráter voluntário e do sigilo referentes à sua identificação. $\mathrm{O}$ número de participantes foi estabelecido segundo o critério de saturação definido por Sá (1998). De acordo com este critério, as entrevistas podem ser encerradas no momento em que os conteúdos temáticos começarem a se repetir e não surgirem elementos novos ou diferenciados nas falas dos participantes.

\section{Procedimentos de análise dos dados}

As entrevistas foram gravadas e transcritas na íntegra. Os conteúdos apreendidos foram processados pelo software Alceste (Analyse Lexicale par Contexte d'un Ensemble de Segments de Texte), versão 4.9, que realizou a análise lexical, permitindo a visualização da classificação hierárquica descendente e ascendente (Ribeiro, 2004).

Este software foi elaborado por Reinert, em 1986, e tem por finalidade realizar uma série de procedimentos estatísticos aplicados a bancos de dados textuais, que podem ser originados de entrevistas, diálogos, obras literárias, artigos de jornais e revistas, entre outros. O programa apoia-se em cálculos efetuados sobre a coocorrência de palavras nos segmentos de texto, buscando distinguir classes de palavras que representem formas distintas de discurso sobre o tópico de interesse da investigação, neste caso específico, a IRC (Saraiva, Coutinho \& Miranda, 2011).

Os dados dos questionários foram processados por meio do Statistical Package for Social Science, versão 19.0 (SPSS-19.0), e analisados a partir da estatística descritiva, observando-se a frequência, a porcentagem, a média e o desvio padrão. A análise dos dados provenientes da HADS-D seguiu as indicações de Botega e cols. (1995), considerando-se o ponto de corte " 8 " como indicativo de presença de sintomatologia depressiva.

\section{Resultados}

Os resultados advindos da HADS-D, aplicada com a finalidade de rastrear a depressão nos pacientes em tratamento de hemodiálise, registraram que $20 \%$ dos participantes apresentaram a depressão, com uma pontuação que variou de 8 a 18 pontos $(M=5,42$; $\mathrm{DP}=3,9)$.

Os dados oriundos da entrevista e processados pelo software Alceste, originou um corpus constituído de 26 unidades de contexto iniciais (UCIs), com 20.057 ocorrências, das quais 2.440 palavras diferentes e média de 4 ocorrências por palavra, com frequência igual ou superior à média e $\chi^{2} \geq 3,84(p<0,05)$. Ao reduzir o vocabulário às suas raízes, foram encontrados 422 radicais analisáveis e 436 Unidades de Contexto Elementares (UCEs). A Análise Hierárquica Descendente reteve $69 \%$ do total das UCEs do corpus, as quais foram organizadas em cinco classes, conforme pode ser visto na Figura 1.

No dendrograma observa-se, em uma primeira divisão, que o corpus originou dois subcorpora, um deles aglutinou a classe 2, denominada "aspectos relacionados à nutrição", e o outro foi subdividido em dois novos subcorpora. Um deles aglomerou a classes 1 "significados da hemodiálise" e a classe 5 , "suporte familiar e estrutural", resultando em um campo conceituado, "hemodiálise e suporte no tratamento da IRC". O outro subcorpora resultou na junção da classe 3 "descoberta do diagnóstico" e da classe 4 definido "causas e sintomas da IRC", constituindo o campo "diagnóstico, causas e sintomas da IRC".

A classe 1, "Significados da hemodiálise", foi constituída por 74 UCEs, representando $25 \%$ do total do corpus, sendo composta por palavras e radicais no intervalo entre $\chi^{2}=19$ (ajuda) e $\chi^{2}=4$ (acha). As variáveis-atributos que mais contribuíram para esta classe foram os pacientes com ensino superior, sem depressão e com idade entre 26 e 33 anos. A seguir, estão listadas algumas UCEs que justificam a denominação desta classe:

[...] o dia de tratamento era o pior dia da minha vida, mas
depois eu fui vendo que a máquina só me trazia benefícios;
[...] o tratamento significa tudo de bom; eu acho que o tra-
tamento é uma benção de Deus; a hemodiálise ajuda uma
parte do seu corpo; esse tratamento é uma coisa que não
esquenta a minha cabeça; mas Deus tem me ajudado e eu
tenho suportado.

A classe 5, "suporte familiar e estrutural", foi composta por 72 UCEs, correspondendo a $24 \%$ do corpus, sendo formada por palavras e radicais no intervalo entre $\chi^{2}=25$ (vai) e $\chi 2=9$ (sair). As variáveis descritivas que mais contribuíram para esta classe foram: ter idade entre 34 e 41 anos; e possuir o ensino fundamental. A seguir, estão listadas algumas UCEs representativas 


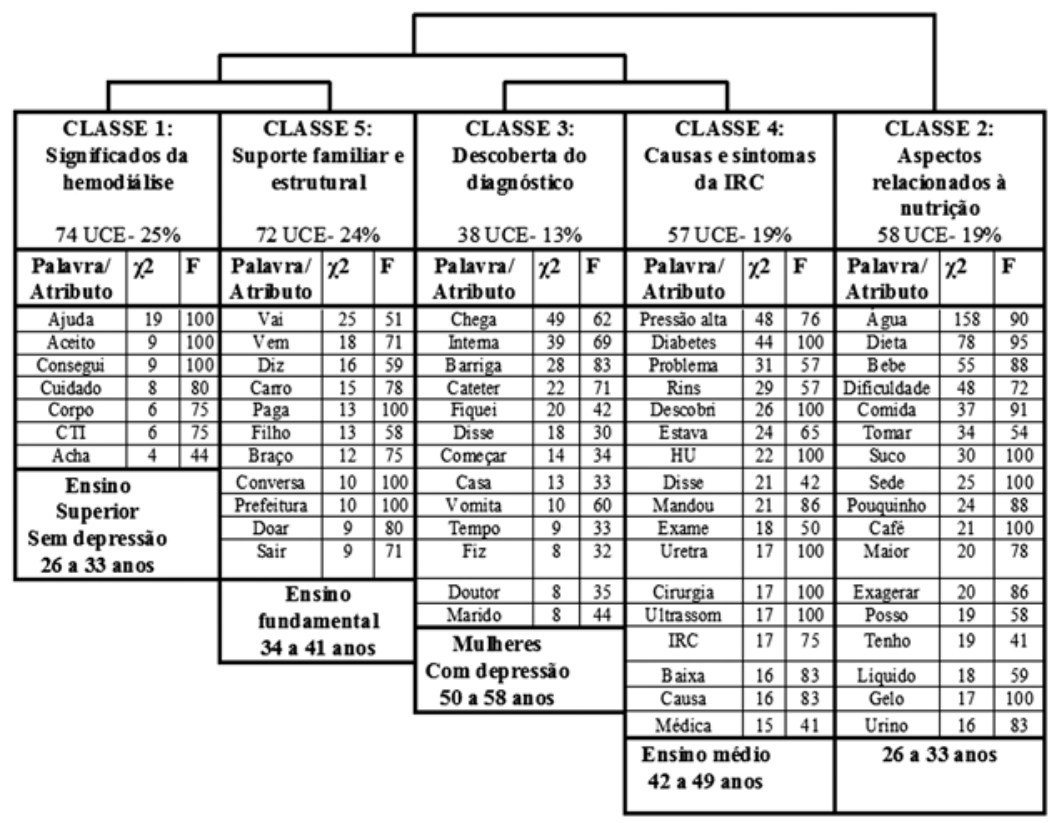

Figura 1. Dendrograma da distribuição de classes e variáveis resultantes da análise Alceste $(\mathrm{N}=26)$.

desta classe, que trata do apoio dado pela família e por instâncias governamentais aos pacientes em tratamento de hemodiálise:

[...] eu ligo pra um filho meu vem numa moto pra mim pegar e vai buscar uma medicação ali; eu moro em Santa Rita, o carro demora muito para vir me pegar, todo mundo vai embora e eu fico lá na frente sozinha esperando esse carro; meu filho quem vai doar o rins pra mim; porque a gente tinha que pagar carro, era uma dificuldade, ai a gente conseguiu aqui no [hospital], meu filho veio e conseguiu porque a prefeitura liberou um carro pra mim.

A classe 3, intitulada "descoberta do diagnóstico", formada por 38 UCEs, contabilizou $13 \%$ do total do corpus. Foi composta por palavras e radicais no intervalo entre $\chi^{2}=49$ (chega) e $\chi^{2}=8$ (marido), revelando o discurso dos pacientes do sexo feminino, com idade entre 50 e 58 anos, que apresentavam a sintomatologia depressiva. A seguir, são listadas algumas pseudofrases que constituíram esta classe, a qual abordou os sentimentos e pensamentos vivenciados no momento em que os pacientes receberam o diagnóstico de IRC:

[...] quando cheguei aqui [hospital] já colocou o cateter no men pescoco e comecei a dialisar; me deu muita agonia; fiquei nervosa com medo, botei pra chorar [quando recebi o diagnóstico]; tinha muito medo de morrer; me recusei a colocar o cateter durante 22 dias; a doutora me disse você vai ficar internada pra fazer uns exames, mas eu já sabia o que ela ia fazer.

A classe 4, conceituada de "causas e sintomas da IRC", que conteve 57 UCEs, correspondendo a $19 \%$ do total do corpus, foi composta por palavras e radicais no intervalo entre $\chi^{2}=48$ (pressão alta) e $\chi^{2}=15$ (médico). As variáveis-atributos que mais contribuíram para esta classe foram os pacientes renais com ensino médio e com idade entre 42 e 49 anos. As interlocuções a seguir exemplificam alguns sintomas e causas por eles atribuídos à doença:

\begin{abstract}
[...] quando eu cheguei lá a pressão estava muito alta, ai ele mandou bater um ultrassom das vias urinarias, ai constatou que os meus dois rins tinham diminuido; eu era diabética. Não sabia que a diabetes causava um problema renal; en estava sentindo dificuldades para urinar, com a uretra inflamada; descobriram que eu tinha cálculo renal e foi através disso que disseram que os rins estavam paralisando; então a diabetes foi e pegou os rins; tava sentindo umas dores, febre, dor de cabeça, vômito. Eu não sabia que era problema renal.
\end{abstract}

Por fim, a classe 2, categorizada de "aspectos relacionados à nutrição", conteve 58 UCEs, expressando $19 \%$ do corpus. Foi composta por palavras e radicais no intervalo entre $\chi^{2}=158$ (água) e $\chi^{2}=16$ (urino), sendo descrita por participantes com idade entre 26 e 33 anos. Em seguida, aparecem algumas UCEs representativas 
desta classe, as quais revelam a maneira como os atores sociais lidam com as restrições alimentares provocadas pela IRC:

[...] não pode comer muita coisa que aumente o potássio, o fósforo; não pode nem tocar em jaca, nem em carambola; água ainda eu tomo, liquido em geral, café eu sempre exagero; não faco dieta, como tudo, bebo um copão cheio de água; estou passando uma sede; evito muito sal, carne de porco; fazer bochecha com água na boca e jogar fora; não posso beber água, se beber incha o rosto e os pés; eu não obedeço muito a dieta não.

Para complementar os resultados obtidos por meio da CHD, o ALCESTE realizou a Análise Fatorial de Correspondência (AFC), resultando no plano fatorial demonstrado na Figura 2. Tal figura demonstra o traçado de dois eixos, que juntos explicaram $64 \%$ da variância total das UCEs. O conteúdo das falas dos participantes distribui-se em cinco zonas ou conglomerados, de modo não aleatório e correspondente às formas específicas das classes.

$\mathrm{Na}$ linha horizontal, o eixo 1 revela as maiores cargas fatoriais identificadas no Alceste, explicando 37\% da variância total das UCEs. Nesse eixo, no lado negativo ou à esquerda da Figura 2, destacam-se as palavras aglutinadas na classe 3 (cheguei, interna, barriga, cateter, fiquei), apreendidas no discurso dos participantes do sexo feminino, com idade entre 50 e 58 anos e com sintomas depressivos. Por oposição, no mesmo eixo, no lado positivo, à direita, posicionam-se as palavras com maiores cargas fatoriais agrupadas na classe 5 (vai, vem, digo e vim), que emergiram dos pacientes com ensino fundamental e com idade entre 34 e 41 anos de idade, e na classe 2 (com, dificuldade, água, dieta, bebo), formuladas pelos pacientes com idade entre 26 e 33 anos.

Com relação ao eixo 2, na linha vertical da Figura 2, destacam-se dois agrupamentos de palavras, que explicam $27 \%$ da variância total das UCEs. Nele emergiram dois campos semânticos: um, no plano superior, com as contribuições dos pacientes com ensino superior e sem sintomas depressivos, da classe 1 (ajuda, máquina, significa, vida e tratamento); e, por contraste, no plano inferior, posicionam-se as palavras oriundas dos participantes com ensino médio e com idade entre 42 e 49 anos, categorizadas na classe 4 (rins, diabetes, pressão alta e problema).

Nos resultados da AFC sobressaem os espaçamentos ou distâncias euclidianas entre as cinco classes, dispostas no plano fatorial. Assim, pode-se perceber o distanciamento do discurso dos pacientes com sintomas depressivos, que foram mais expressivos na classe 3 e estiveram voltados para o impacto do recebimento do diagnóstico. Em contrapartida, na classe 1 encontra-se

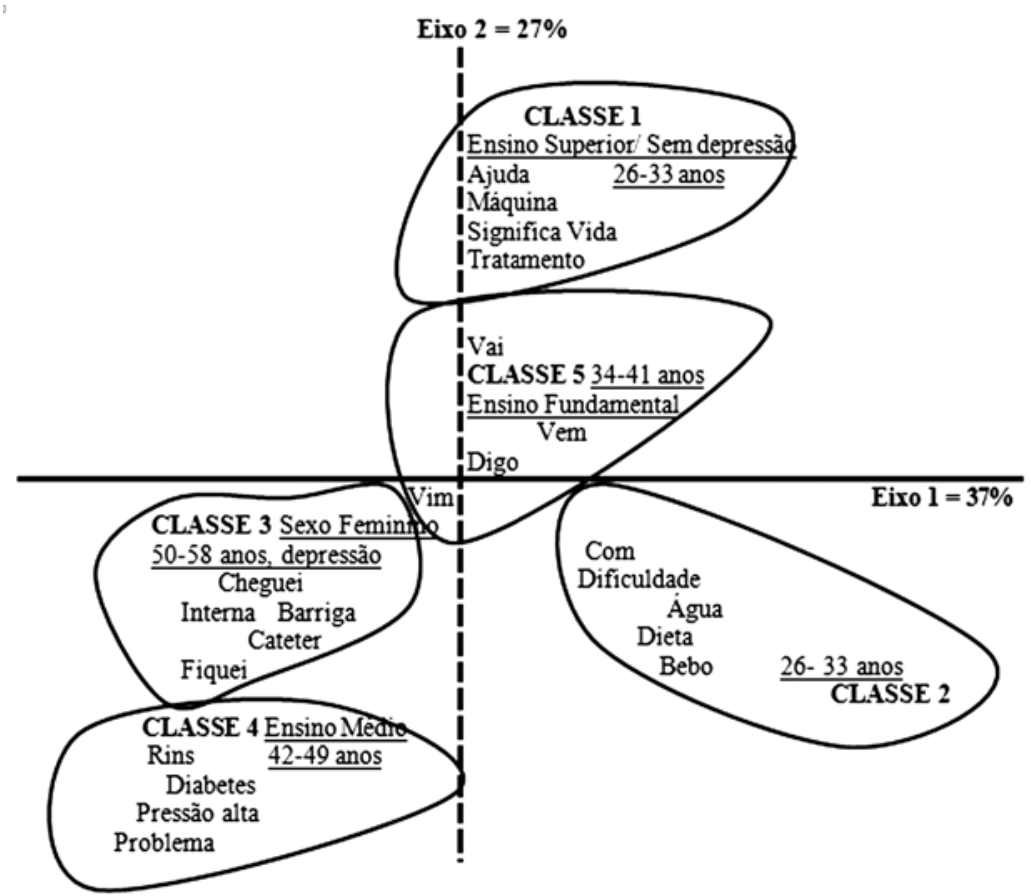

Figura 2. Análise Fatorial de Correspondência gerada pelo ALCESTE. 
o discurso dos pacientes sem a sintomatologia depressiva, os quais se referiram ao apoio recebido pela família, demonstrando que este é um fator de proteção e melhoria da qualidade de vida no contexto dessa síndrome.

\section{Discussão}

Pode-se perceber que o percentual de depressão constatado neste estudo se situou no intervalo das prevalências apontadas pela literatura. Conforme Almeida (2003), dependendo dos instrumentos utilizados, percebe-se uma diferença na variação dos índices de prevalência da depressão no contexto da hemodiálise. Estudos utilizando o Inventário para Depressão de Beck encontraram maiores índices de depressão em suas amostras, Costa e cols. (no prelo) constataram uma prevalência de $56,3 \%$ e Cukor e cols. (2006) relataram que $42 \%$ dos pacientes em hemodiálise sofrem de depressão.

Os índices mencionados revelam que os profissionais da saúde devem estar atentos para a comorbidade da depressão nesse contexto, uma vez que essa realidade implica negativamente na qualidade de vida, na diminuição da imunidade e da capacidade funcional, no relaxamento dos cuidados pessoais e na adesão aos tratamentos e dietas. A síndrome também parece estar relacionada à intensificação de problemas, tanto financeiros e profissionais quanto familiares, e à maior possibilidade do abuso ou dependência de álcool e outras drogas. Consequentemente, esses problemas acarretam o aumento do número de consultas ambulatoriais, internações e até mortalidade (Chilcot e cols., 2008; Cukor, Coplan, Brown, Peterson, \& Kimmel, 2008).

Tomando-se como referência o dendrograma das cinco classes (Figura 1) e o plano fatorial de correspondência (Figura 2), foi possível perceber RS acerca da IRC elaborada por pacientes com e sem depressão no contexto da hemodiálise. Conforme se pode observar na classe 3 do dendrograma, os pacientes com a síndrome depressiva ancoraram as suas representações acerca da IRC na descoberta do diagnóstico. Para este grupo, o conhecimento do diagnóstico foi permeado por sentimentos de agonia, nervosismo, medo da morte, choro e recusa em iniciar o tratamento. $\mathrm{O}$ impacto ao saber sobre a IRC pode gerar sentimentos de tristeza e desencadear quadros psicopatológicos importantes, como é o caso da depressão. A literatura é consensual ao apontar a depressão como um dos transtornos psicoafetivos mais comuns em pacientes sob tratamento de hemodiálise (Chilcot e cols., 2008; Cohen, Norris, Acquaviva, Peterson, Kimmel, 2007). Para Pereira e Guedes (2009), a experiência de receber o diagnóstico de doença renal crônica pode ser descrito, em muitos casos, como um dos momentos mais difíceis para o paciente.

Já os pacientes sem a sintomatologia depressiva destacaram, em suas representações sociais da IRC, elementos associados ao significado positivo do tratamento, conforme é possível observar na classe 1 do dendrograma. Nesta classe, os participantes ancoraram a hemodiálise como algo positivo e benéfico, que lhes garante sobrevivência e maior longevidade. Apesar disso, na sua fase inicial o tratamento foi objetivado como um dos piores dias para esses pacientes. Observou-se também a confiança em Deus, como fonte de suporte espiritual para o enfrentamento dessa fase de suas vidas.

Segundo Pereira e Guedes (2009) e Machado e Car (2003), a hemodiálise é o principal fator para a sobrevivência dos pacientes com doença renal em estágio avançado. Portanto, torna-se necessária para a manutenção do bem-estar dessas pessoas, apesar de lhes trazer uma realidade sofrida.

Outros autores também observaram que a hemodiálise foi representada com base em sentimentos ambíguos de amor e ódio por pessoas que precisam deste tratamento para sobreviver, uma vez que se trata de uma terapêutica que lhes assegura a vida, mas torna-as dependentes da tecnologia. Por causa disso, identifica-se, entre os elementos simbólicos atribuídos à hemodiálise, um binômio que expressa a relação ambivalente de vida e morte, presente em seu cotidiano (Reis, Guirardello \& Campos, 2008).

O estudo realizado por Campos e Turato (2010) reafirma a condição essencial e exclusiva da hemodiálise, enquanto forma de tratamento para a IRC. Para os participantes da pesquisa, a hemodiálise foi vinculada à sobrevivência e à obrigação, uma vez que a IRC é uma doença marcada pela falta de outras opções de terapia e a realização desse procedimento garante que o paciente tenha suas funções renais estabilizadas.

O apego a Deus, como fonte de suporte espiritual, possibilidade de fortalecimento e superação na vida dos pacientes que participaram do presente estudo, coaduna-se com o estudo de Pereira e Guedes (2009). Percebeu-se consenso entre as RS da IRC dos pacientes com e sem depressão, no tocante ao suporte familiar e estrutural, às causas e sintomas da IRC e aos aspectos relativos às restrições alimentares. 
Conforme observado na classe 5, "suporte familiar e estrutural", a família foi apontada como um baluarte no acompanhamento aos locais de tratamento, no auxílio financeiro para a compra de medicamentos e na doação de rins destinada a transplantes dos pacientes. $\mathrm{O}$ apoio institucional foi mencionado no que se refere ao acesso dos pacientes aos locais de tratamento por meio de carros disponibilizados por prefeituras. Percebeu-se que a família surgiu como apoio principal para ajudar a enfrentar as dificuldades, pois são os membros familiares que estão próximos e buscam ajudar em todos os momentos, fazendo com que os pacientes lutem, sejam otimistas e não se sintam sozinhos (Pereira \& Guedes, 2009; Pietrovski \& Dall'Agnol, 2006).

Em um estudo de caso com paciente renal, realizado por Dyniewicz, Zanella e Kobus (2004), a categoria de "apoio familiar" também esteve presente. A família foi definida como um bem maior, onde há uma convivência harmoniosa, voltada para a frequente demonstração de afeto mútuo, ao mesmo tempo em que se compartilham as preocupações do dia a dia.

Conforme Patat, Stumm, Kirchner, Guido e Barbosa (2012), o fato de os pacientes renais possuírem uma estrutura de apoio afetivo (serem casados, possuírem filhos ou residirem com companheiros) pode ajudá-los a enfrentar a doença crônica. $\mathrm{O}$ convívio e o apoio familiar constituem um suporte que suaviza os danos físicos, psicológicos e socioeconômicos provocados no indivíduo pela doença e pelo tratamento.

O apoio estrutural foi outro fator que sobressaiu na fala dos atores sociais da presente pesquisa. Provavelmente, para os pacientes com grau de escolaridade correspondente ao ensino fundamental e com idades entre 34 e 41 anos, muitas atividades econômicas tornam-se comprometidas, em função do tempo gasto para a realização da hemodiálise e das limitações que são peculiares a essa modalidade terapêutica. Nesse caso, passam a depender dos benefícios de programas assistenciais ou de serviços prestados por prefeituras, nas cidades onde moram (Centenaro, 2010). É comum que pacientes com IRC não possuam atividades remuneradas e necessitem de apoio financeiro de familiares e/ou do estado (Gonçalves, 2012; Lanza, Chaves, Garcia, \& Brandão, 2008).

De acordo com a classe 4, os pacientes com idade entre 42 e 49 anos e com o ensino médio verbalizaram sobre as causas e os sintomas da IRC. As principais objetivações desta classe foram: pressão alta, diabetes, dificuldade para urinar, uretra inflamada, dor, febre e vômito.
As pessoas podem sobreviver com apenas $10 \%$ da função renal, o que contribui para que os sintomas da IRC demorem anos para ser notados. Nesse sentido, devido ao quadro clínico assintomático da doença, os indivíduos desconhecem a sua existência até que ela atinja um estágio bastante avançado, percebendo-a apenas quando cerca de $50 \%$ da função renal já foi perdida (Ribeiro e cols., 2008; Smeltzer \& Bare, 2009).

Para Daugirdas, Blake e Ing (2010), o sofrimento do doente renal é mais intenso no momento do recebimento da notícia de perda da função dos rins. Uma vez que a doença renal crônica não apresenta sinais ou sintomas que alertem para o início do problema, as pessoas não sentem dor, continuam urinando e, portanto, com a falsa impressão de que seus rins estão funcionando.

A ocorrência da IRC verbalizada pelos participantes desta pesquisa coaduna-se com os estudos de Fayer (2010), Gonçalves (2012), Kruger (2011) e Pereira e Guedes (2009). Como mostram as verbalizações, o adoecimento foi representado como um momento inesperado, que causa surpresa devido à falta de sintomas perceptíveis da sua fase inicial. Os participantes revelaram, ainda, o desconhecimento dos aspectos básicos da terapia hemodialítica, ao iniciarem o tratamento.

$\mathrm{Na}$ classe 2, "aspectos relacionados à nutrição", constataram-se as representações dos pacientes referentes às dificuldades enfrentadas para seguirem a dieta alimentar, com a qual devem conviver ao longo da vida em consequência da IRC. Conforme Riella e Martins (2001), um dos grandes desafios para os pacientes em hemodiálise é seguir a dieta oral, especialmente em razão das mudanças nos hábitos alimentares e do padrão comportamental de cada um. Em muitos casos, é necessário restringir a ingestão de seus alimentes favoritos, substituindo-os por outros, não tão agradáveis ao paladar. Uma parte considerável dos participantes da presente pesquisa relatou não seguir a dieta, apesar de saber da importância que ela possui para o tratamento da IRC.

As restrições alimentares e hídricas são fundamentais para o sucesso do tratamento e para o bem-estar do indivíduo portador da IRC. No entanto, geram descontentamento e não aceitação, por imporem privações severas, que envolvem até mesmo restrições sobre a quantidade de água a ser ingerida pelo paciente. Com isso, o tratamento é permeado por consequências, que tendem a ser consideradas como difíceis, árduas e repletas de restrições.

$\mathrm{O}$ ato de comer está relacionado com um sistema de valores que, quando alterados, têm repercussão na 
vida social e no próprio significado dessa atividade para o indivíduo. Esses aspectos transcendem o lado meramente terapêutico da dieta e promovem uma interação entre o comer e a experiência da doença (Reis e cols., 2008).

A partir da análise padrão do ALCESTE e da análise fatorial de correspondência, observou-se o conteúdo das RS acerca da IRC elaboradas pelos participantes da pesquisa. De modo geral, observou-se que o conhecimento do senso comum dos pacientes renais foi associado ao suporte familiar e espiritual, aos fatores nutricionais e ao descobrimento do diagnóstico. Tais representações encontram-se relacionadas com as adversidades psicossociais vivenciadas, no contexto da doença renal, por esses grupos de pertença.

\section{Considerações finais}

Com o objetivo principal de apreender as representações sociais acerca da IRC e do tratamento elaboradas por pacientes nefrológicos com e sem depressão em tratamento da hemodiálise, o presente estudo permitiu o resgate do saber do senso comum, com a evidência das diferentes concepções, sentimentos, atributos e imagens que permearam toda a construção deste conhecimento. O reconhecimento da importância do conhecimento espontâneo leva a um novo contorno de compreensão dessas doenças, de modo a transcender perspectivas medicalizantes, tornando possível o entrelaçamento de significados do ser humano enquanto ser multifacetado, permeado de aspectos sociais, biológicos, afetivos e psicológicos.

De modo geral, observou-se que o conhecimento do senso comum dos pacientes renais foi associado ao suporte familiar e espiritual, aos fatores nutricionais e ao descobrimento do diagnóstico. Paralelamente, foi possível mensurar o índice da depressão nos pacientes nefrológicos por meio dos resultados da subescala hospitalar de depressão. Este instrumento evidenciou que $20 \%$ dos pacientes apresentaram a sintomatologia depressiva, uma prevalência que aponta para a necessidade de atenção para esse tipo de comorbidade no tratamento da IRC.

A utilização de uma amostra não probabilística revela-se como uma importante limitação no que diz respeito à possibilidade de generalização dos resultados. Sendo assim, sugere-se cautela ao comparar estes resultados a de outros autores. Como esta pesquisa foi realizada somente em instituições públicas, ela não possibilitou observar se existiam diferenças entre o contexto público e o privado. Destaca-se também que a prevalência da depressão encontrada não se trata de um diagnóstico, uma vez que se utilizou apenas um instrumento para o rastreamento da depressão, e que para diagnosticar tal fenômeno seria necessária uma bateria de instrumentos.

No que se refere à aplicabilidade deste estudo, espera-se que seus resultados possam assinalar caminhos para futuras investigações, principalmente no que se refere às implicações dos aspectos psicossociológicos na manifestação e tratamento da IRC. Acredita-se, também, que os resultados podem subsidiar a implementação de programas de saúde pautados em uma abordagem interdisciplinar que enfoque o suporte psicossociológico e nutricional no tratamento da doença renal, uma vez que o momento do diagnóstico e a relação com a alimentação emergiram como aspectos relevantes nesse processo.

\section{Referências}

Almeida, A. M. (2003). Revisão: a importância da saúde mental na qualidade de vida e sobrevida do portador de insuficiência renal crônica, Jornal Brasileiro Nefrologia, 25(4), 209-214.

Botega, N. J., Bio, M. R., Zomignani, M. A., Garcia Jr, C., \& Pereira, W. A. B. (1995). Transtornos do humor em enfermaria de clínica médica e validação de escala de medida (HAD) de ansiedade e depressão. Revista Saúde Pública, 29(5), 359-363.

Brasil. Ministério da Saúde. CNS. (1996). Resolução 196/96 sobre pesquisa envolvendo seres humanos. Brasília: Ministério da Saúde.

Campos, C. J. G. \& Turato, E. R. (2010). Tratamento hemodialítico sob a ótica do doente renal: estudo clínico qualitativo. Revista Brasileira de Enfermagem, 63(5), 799-805.

Castro, M. M. C., Quarantini, L., Batista-Neves, S., Kraychete, D. C., Daltro, C. C., \& Miranda-Scippa, A. (2006). Validade da Escala Hospitalar de Ansiedade e Depressão em pacientes com dor crônica. Revista Brasileira de Anestesiologia, 56(5), 470-477.

Centenaro, G. A. (2010). A intervenção do serviço social ao paciente renal crônico e sua família. Ciência \& Saúde Coletiva, 15(1), 1881-5.

Chilcot, J., Wellsted, D., Silva-Gane, M., \& Farrington, K. (2008). Depression on dialysis. Nephron Clinical Practice, 108(4), 256-264. 
Cohen, S. D., Norris, L., Acquaviva, K., Peterson, R. A., \& Kimmel, P. L. (2007). Screening, diagnosis, and treatment of depression in patients with end-stage renal disease. Clinical Journal of the American Society of Nephrology, 2(6), 1332-1342.

Costa, F. G., Coutinho, M. P. L., Melo, J. R. F., \& Oliveira, M. X. (no prelo). Rastreamento da depressão no contexto da insuficiência renal crônica. Revista Temas em Psicologia.

Coutinho, M. P. L. (2005). Depressão infantil e representações sociais ( $2^{\mathrm{a}}$ ed.). João Pessoa: Editora Universitária/ UFPB.

Coutinho, M. P. L. \& Saraiva, E. R. A. (2013). Teoria das representações sociais. Em N. T. Alves (Orgs.), Psicologia: reflexões para ensino e extensão. (pp. 73-114). João Pessoa, PB: Editora Universitária.

Cukor, D., Coplan, J, Brown, C., Peterson, R. A., \& Kimmel, P. L. (2008). Course of depression and anxiety diagnosis in patients treated with hemodialysis: a 16-month follow-up. Clinical Journal of American Society of Nephrology, 3(6), 1752-1758.

Cukor, D., Peterson, R. A., Cohen, S. D., \& Kimmel, P. L. (2006). Depression in end-stage renal disease hemodialysis patients. Nature Clinical Practice Nephroogyl, 2(12), 678-687.

Daugirdas, J. T., Blake, P. G., \& Ing, T. S. (2010). Manual de diálise ( $4^{\mathrm{a}}$ ed.). Rio de Janeiro: Guanabara.

Dyniewicz, A. M., Zanella, E., \& Kobus, L. S. G. (2004). Narrativa de uma cliente com insuficiência renal crônica: a história oral como estratégia de pesquisa. Revista Eletrônica de Enfermagem, 6(2), 199-212.

Fayer, A. A. M. (2010). Repercussões psicológicas da doença renal crônica: comparação entre pacientes que iniciam o tratamento hemodialítico após ou sem segmentos nefrológicos prévios. (Dissertação de Mestrado), Programa de Pós-Graduação em Medicina, Universidade de São Paulo, SP.

Ferreira, P. L. \& Anes, E. J. (2010). Medição da qualidade de vida de insuficientes renais crônicos: criação da versão portuguesa do KDQOL-SF. Revista Portuguesa de Saúde Pública, 28(1), 31-39.

Finger, G., Pasqualotto, F. F., Marcon, G., Medeiros, G. S., Abruzzi Junior, J., \& May, W. S. (2011). Sintomas depressivos e suas características em pacientes submetidos à hemodiálise. Revista da Associação Médica do Rio Grande do Sul, 55(4), 333-38.
Garcia, T., Veiga, J. P. R., \& Motta, L. O. C. (2010). Comportamento depressivo e má qualidade de vida em homens com insuficiência renal crônica, submetidos à hemodiálise. Revista Brasileira de Psiquiatria, 32(4), 369-374.

Gonçalves, C. S. (2012). As representações sociais sobre a doença renal crônica. (Dissertação de Mestrado), Programa de Pós-Graduação em Enfermagem, Universidade Federal do Paraná, Curitiba, PR.

Kimmel, P. L., Cohen, S. D., \& Peterson, R. A. (2008). Depression in patients with chronic renal disease: where are we going? Journal of Renal Nutrition, 18(1), 99-103.

Kruger, T. S. (2011). Adesão ao tratamento em pacientes renais crônicos: uma revisão integrativa. (Monografia de Conclusão de Curso), Universidade Federal do Rio Grande do Sul, Porto Alegre, RS.

Lanza, A. H. B., Chaves, A. P. A., Garcia, R. C. P., \& Brandão, J. A. G. (2008). Perfil biopsicossocial de pacientes renais crônicos em tratamento hemodialítico. Arquivos Brasileiros de Ciências da Saúde, 33(3), 141-145.

Lima, A. F. C. \& Gualda, D. M. R. (2000). Reflexões sobre a qualidade de vida do cliente renal crônico submetido à hemodiálise. Revista Nursing, 3(30), $20-23$.

Marcolino, J. A. M, Mathias, L. A. S. T, Piccinini Filho, L., Guaratini, A. A., Suzuki, F. M., \& Alli, L. A. C. (2007). Escala Hospitalar de Ansiedade e Depressão: estudo da validade de critério e da confiabilidade com pacientes no pré-operatório. Revista Brasileira de Anestesiologia, 57(1), 52-62.

Machado, L. R. C. \& Car, M. R. (2003). A dialética da vida cotidiana de doentes com insuficiência renal crônica: entre o inevitável e o casual. Revista da Escola de Enfermagem da USP, 37(3), 27-35.

Moscovici, S. (2011). Representações sociais: investigações em psicologia social ( $7^{\mathrm{a}}$ ed.). Petrópolis, RJ: Vozes.

Nifa, S. \& Rudnicki, T. (2010). Depressão em pacientes renais crônicos em tratamento de hemodiálise. Revista da Sociedade Brasileira de Psicologia Hospitalar, 13(1), 64-75.

Patat, C. L., Stumm, E. M. F., Kirchner, R. M., Guido, L. A., \& Barbosa, D. A. (2012). Análise da qualidade de vida de usuários em hemodiálises. Enfermería Global, 11(27), 54-65.

Psico-USF, Bragança Paulista, v. 19, n. 3, p. 387-398, set./dez. 2014 
Pietrovski, V. \& Dall'Agnol, C. M., (2006). Situações significativas no espaço-contexto da hemodiálise: o que dizem os usuários de um serviço? Revista Brasileira de Enfermagem, 59(5), 630-5.

Pereira, L. P. \& Guedes, M. V. C. (2009). Hemodiálise: a percepção do portador renal crônico. Cogitare Enfermagem, 14(4), 689-95.

Ramos, I. C., Queiroz, M. V. O., \& Jorge, M. S. B. (2008). Cuidado em situação de doença renal crônica: representações sociais elaboradas por adolescentes. Revista Brasileira de Enfermagem, 61(2), 193-200.

Reis, C. K., Guirardello, E. B., \& Campos, C. J. O. (2008). O indivíduo renal crônico e as demandas de atenção. Revista Brasileira de Enfermagem, 61(3), 336-341.

Ribeiro, A. S. M. (2004). Alceste: análise quantitativa de dados textuais. Manual não publicado. Laboratório de Psicologia do Desenvolvimento Social. Brasília: Instituto de Psicologia, UNB.

Ribeiro, R. C. H. M., Oliveira, G. A. S. A., Ribeiro, D. F., Bertolin, D. C., Cesarino, C. B., Lima, L. C. E. Q., \& Oliveira, S. M. (2008). Caracterização e etiologia da insuficiência renal crônica em unidade de nefrologia do interior do Estado de São Paulo. Acta Paulista de Enfermagem, 21(Especial-Nefrologia), 207-211.
Riella, M. C. \& Martins, C. (2001). Nutrição e o rim. Rio de Janeiro: Guanabara Koogan.

Romão Junior, J. E. (2007). A doença renal crônica: do diagnóstico ao tratamento. Prática Hospitalar, 52, 183-187.

Sá, C. P. (1998). A construção do objeto de pesquisa em representaçoes sociais. Rio de Janeiro, RJ: UERJ.

Saraiva, E. R. A., Coutinho, M. P. L., \& Miranda, R. S. (2011). O emprego do Software Alceste e o desenvolvimento do mundo lexical em pesquisa documental. Em M. P. L. Coutinho \& E. R. A. Saraiva (Orgs.). Métodos de pesquisa em psicologia social: perspectivas qualitativas e quantitativas (pp. 67-94). João Pessoa, PB: Editora Universitária.

Smeltzer, S. C. \& Bare, B. G. (2009). Tratado de enfermagem médico-cirúrgica. (J. E. F., Cruz, Trad., 11ª ed.). Rio de Janeiro, RJ: Guanabara Koogan.

Thomas, C. V. \& Alchieri, J. C. (2005). Qualidade de vida, depressão e características de personalidade em pacientes submetidos à hemodiálise. Avaliação Psicológica, 4(1), 57-61.

Recebido em: 13/12/2013

Reformulado em: 25/03/2014

Aprovado em: 16/04/2014 
Sobre as autoras:

Fabrycianne Gonçalves Costa é graduada em Licenciatura em Psicologia (2006), Formação em Psicologia (2007) e especialização em Neuropsicologia (2009) pelo Centro Universitário de João Pessoa. É mestre em Psicologia Social pela Universidade Federal da Paraíba - UFPB (2013) e doutoranda em Psicologia Social pela UFPB.

Maria da Penha de Lima Coutinho é graduada em Psicologia pela Universidade Federal da Paraíba (1978), mestre em Psicologia da Saúde pela Universidade Federal da Paraíba (1986), doutora em Psicologia Clínica pela Universidade de São Paulo (2001) e pós-douto pela Universidade Aberta de Lisboa, PT. É coordenadora do Núcleo de Pesquisa Aspectos Psicossociais de Prevenção de Saúde Coletiva.

Inayara Oliveira de Santana é graduada em Licenciatura em Psicologia pela Universidade Federal da Paraíba (2003) e Formação em Psicologia pela Universidade Federal da Paraíba (2004), mestrado em Psicologia Social pela Universidade Federal da Paraíba (2007). É professora da Universidade Federal do Recôncavo da Bahia (UFRB) e doutoranda da UFPB.

\section{Contato com as autoras:}

Fabrycianne Gonçalves Costa.

Rua Maria Helena Rocha, 113 - ap. 1301, B1 A, Aeroclube CEP: 58036-823. João Pessoa- PB. Fone: (83) 9967-5452.

E-mail: fabrycianne@gmail.com 